\title{
Impact of Artificial Intelligence on Economic Development
}

\author{
Ling Hu, Zhaoyong Chen, Zhao Chen* \\ Guangdong University of Foreign Studies, Guangzhou 510000, Guangdong Province, China \\ *Corresponding author: Zhao Chen, chenzhao2002@126.com
}

\begin{abstract}
The economic growth pattern in China is changing from high-speed development to high-quality development. In order to promote high-quality economic development, it is necessary to promote the transformation and upgrading of all aspects of social reproduction. Artificial intelligence has promoted high-quality economic development in China from the aspects of innovation effect, technology spillover effect, factor improvement effect, and technology optimization effect. However, there are still several problems in China's AI development, such as the lack of core technologies, privacy and security, as well as the lack of high-tech talents. To this end, China should advance basic theoretical research and key generic technology development to improve the self-reliance of science and technology, as well as optimize the development environment of the industry and improve the relevant laws and regulations as well as the ethical norms system. A system to train high-quality personnel and a mechanism that allows personnel flow should be established to promote high-quality economic development.
\end{abstract}

Keywords: Artificial intelligence; High-quality development; Industrial upgrading; Industry optimization

Publication date: October 2021; Online publication: October 29, 2021

\section{Introduction}

China's socialism has stepped into a new era, and its economic growth pattern has also entered a critical period of transition from high-speed development to high-quality development. Global competition in science and technology is becoming more intense and complex. Under the tide of economic globalization, China needs to accelerate the formation of a new development pattern with major domestic cycles as the centerpiece. Therefore, domestic and international double cycles need to mutually reinforce each other. It is necessary to emphasize on the role of science and technology innovation as the impetus for future highquality national economic development. Over the past 40 years, China has seized the opportunity to reform and open, while actively participating in international trades and the value chain of the international division of labor. For a long time, China has relied on extensive economic growth, leading to a series of serious problems in the country. With this in mind, improving the quality of economic development has become the inevitable choice of China's current economic long-term policies. With the current context of representative technologies in the age of technological revolution and industrial transformation, artificial intelligence is at the core of an intelligent economic form of data-driven, human-machine collaboration, cross-border integration, co-creation, and sharing. This also provides an effective way for the transition of China's economic development. According to research (Figure 1), the scale of China's AI industry would exceed 180 billion yuan in 2021 and is expected to exceed 450 billion yuan in 2025 . The compound annual growth rate (CAGR) of AI products from 2021 to 2025 would be $24 \%$. In 2020, the scale of related industries driven by AI would exceed 740 billion yuan and in 2025, it would exceed 1.6 trillion yuan. The CAGR of related industries driven by AI from 2021 to 2025 would be $22 \%$. Under the background of new 
industries, new forms of businesses, and new business models, the growth rate of AI output value and the industrial scale driven by it are remarkable.

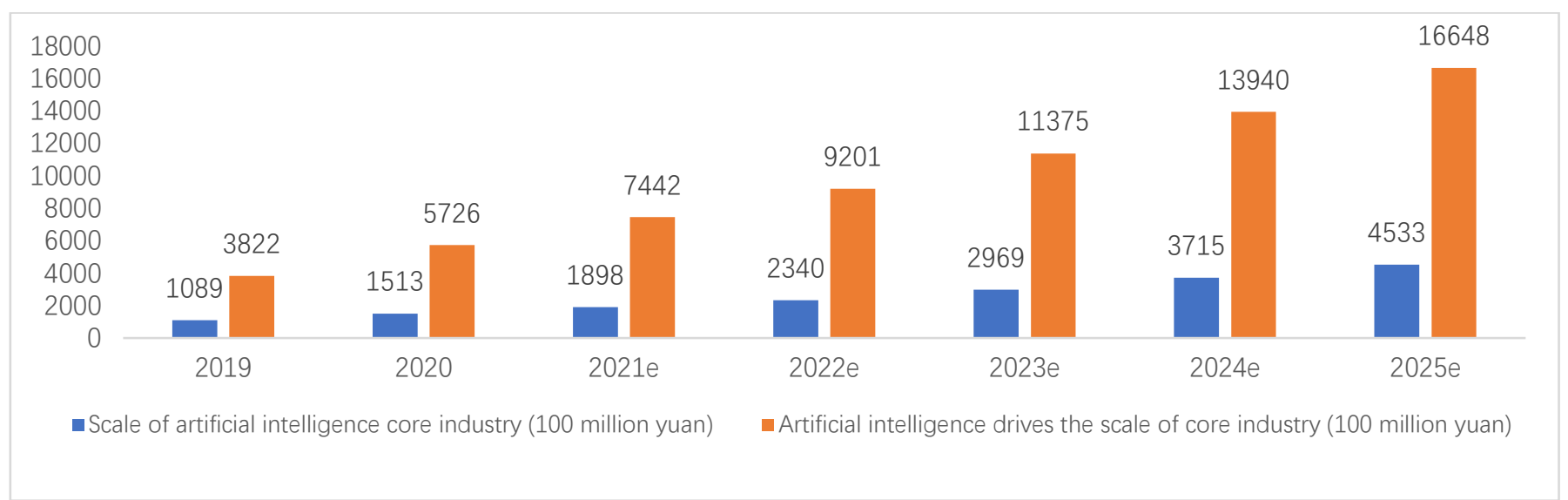

Figure 1. China's artificial intelligence industry and the scale of related industries from 2019 to 2025

Researchers have also conducted relevant studies on the economic development of artificial intelligence. Acemoglu and Restrepo established a production function model from the perspective of technological progress ${ }^{[1]}$. They proposed that the decline of labor population promotes the development of artificial intelligence technology and thus economic growth. By constructing an evolution model of industrial structure that considers artificial intelligence, several researchers regard artificial intelligence as the extension of automation technology and have concluded that artificial intelligence would promote capital to replace labor and improve the productivity of the automation sector ${ }^{[2]}$. In the context of integrating digitalization, intelligence, and networking, the mechanisms and impact of artificial intelligence on China's economy are more profound than those of ordinary technologies. Artificial intelligence can optimize the allocation of industrial resources by driving the science and technology service industry, which constitutes an important support for macro economy ${ }^{[3,4]}$. This study further builds on the ideas of previous literature, analyzes the mechanisms of artificial intelligence to promote high-quality economic growth, and proposes relevant policy suggestions.

\section{Analysis on the mechanisms of artificial intelligence to promote high-quality economic development}

$\mathrm{AI}$ is an important driving force of the new round of scientific and industrial revolution, with a strong spillover effect. China's economy urgently needs to transform to innovation-driven development. Artificial intelligence is characterized by innovation and technological progress. Driven by new theories and technologies, such as 5G, big data, and swarm intelligence, introducing artificial intelligence would have a significant and far-reaching impact on the healthy development of China's social economy.

\subsection{Innovation effect and technology spillover effect of artificial intelligence}

In addition to the characteristics of general-purpose technologies, such as permeability and substitutability, AI technology further includes the characteristics of collaboration and creativity. Among them, synergy refers to the cooperation with various elements of the society to improve economic efficiency, while creativity refers to replacing the human mind with creativity. As the foreword of high technology, artificial intelligence would bring innovation and technological breakthroughs in various fields through the above attributes, directly promoting the progress of science and technology as well as the improvement of productivity. This in turn would trigger the transformation of enterprises, ensuing a shift from labor- 
intensive industries to new innovative industries based on data science. The investment of capital in the field of artificial intelligence can encourage the accumulation of knowledge and experience, which would lead to the improvement of production efficiency, thus producing technological spillover effects. Eventually, it would realize changes in meso and macro dimensions of the law of diminishing marginal effects in order to promote high-speed economic development. The materialized form of knowledge accumulation in artificial intelligence naturally belongs to the innovation of the physical form and is easier applied in social production and life. It is also far beyond the technological innovation and progress of the production mode brought by various non-materialized forms in the past. In addition, as AI is both intelligent and technological, the technological advances it would unleash and the labor force it would bring will be far more significant than the average research and development (R\&D) expenditure.

\subsection{Improvement effect of artificial intelligence}

Artificial intelligence would improve the degree of automation and self-reliance in production and life. Intelligent production equipment would greatly reduce the dependence on [physical] labor, thus triggering the substitution of capital for labor. As AI replaces simple labor, it increases the demand for complex labor which cannot be automated. In other words, AI would change the structures of production, cultivating highend labor and improving the quality as well as quantity of production. Human capital with relevant expertise, skills, and accumulated experience is more productive and adds more value than simple labor. In addition, the emergence of artificial intelligence has given rise to network infrastructure, application infrastructure, and software infrastructure, which have more advantages in productivity compared to traditional public infrastructure. Artificial intelligence also promotes innovation and fosters high-end production factors, which create the conditions for innovating production. In regard to the traditional industry, AI would innovate the classical "demand - design - production - sales - service" model in a more intelligent and efficient way to rearrange each production link.

\subsection{Efficiency optimization effect of artificial intelligence}

AI can improve the income distribution effect by solving the issue of incomplete information. Incomplete information can be countered by obtaining, collecting, and analyzing big data that is randomly generated in the production and life of enterprises and residents via cloud computing and other information technologies. More importantly, artificial intelligence can carry out machine learning on those seemingly irrelevant massive data to discover the rules of social operation, further improve the ability to predict the future, and solve real problems in real time, thus realizing Pareto improvement. The first principle of welfare economics states that the optimal state of perfect competition market is the Pareto equilibrium. In real economy, the allocation efficiency of resources and factors is often reduced due to incomplete information. However, the development of artificial intelligence is able to solve this problem. Artificial intelligence and big data can capture and depict the behavioral characteristics of consumers and solve externalities. Meanwhile, machine learning can solve the resource mismatch problem existing in the current society and promote high-quality economic development.

\section{Development status of artificial intelligence in China}

For a long time, China has struggled to catch-up with the world's science and technology development, and artificial intelligence is one of the few fields that China has taken the lead in the new round of technological revolution. Artificial intelligence also has appeared in all aspects of enterprise production efficiency. In 2020, the scale of China's artificial intelligence industry reached 325.1 billion yuan, and this value is predicted to exceed 450 billion yuan by 2025 . This would also drive China's industrial scale to exceed 1.6 
trillion yuan. AI is accelerating its integration with China's real economy to help transform and upgrade industries as well as improve their quality and efficiency.

According to the Computer Science Rankings (CSRankings) (Figure 2), China currently ranks among the top 200 scientific institutions in the AI category, accounting for $12 \%$ of the world's total, second only to the U.S. Meanwhile, the number of AI companies in China has reached 6,425 at the end of 2020, with the Beijing-Tianjin-Hebei region, the Yangtze River Delta, and the Greater Bay Area accounting for more than $80 \%$ of the total number of companies. Guangdong, Beijing, Shanghai, and Zhejiang are leading the country in terms of the number of enterprises' patent applications. Guangdong, Jiangsu, Beijing, and Zhejiang are the most active regions in the transfer of artificial intelligence patents. These regions have created a new impetus in regional economic development through the supply of scientific and technological achievements. In 2019, the ranking of artificial intelligence in paper publication saw Beijing, Jiangsu, Guangdong, and Hubei taking the lead. In 2020, more than 600,000 people were employed in the field of artificial intelligence. In addition, Beijing, Shanghai, Shenzhen, and Hangzhou were considered homes to more than $80 \%$ of AI talents. According to the Institute of Information and Communication, China bases intelligent tasks on vision, speech, etc. China currently holds a leading role at the present stage of project implementation, but the optimization algorithm model of secondary innovation still needs to be thoroughly improved. Although it is basic in theory, the original model is still seen as disrupted. Errors occur in step technology fields as these still do not have the ability to lead in comparison and require more time to mature. To be specific, there are a large number of AI research and development institutions in China that are committed to absorbing artificial intelligence algorithm technology in the field of transformation and training in scene application. While completing engineering implementation, they only have the ability to carry out minor innovations and optimizations of technical models based on rich scene and user data.

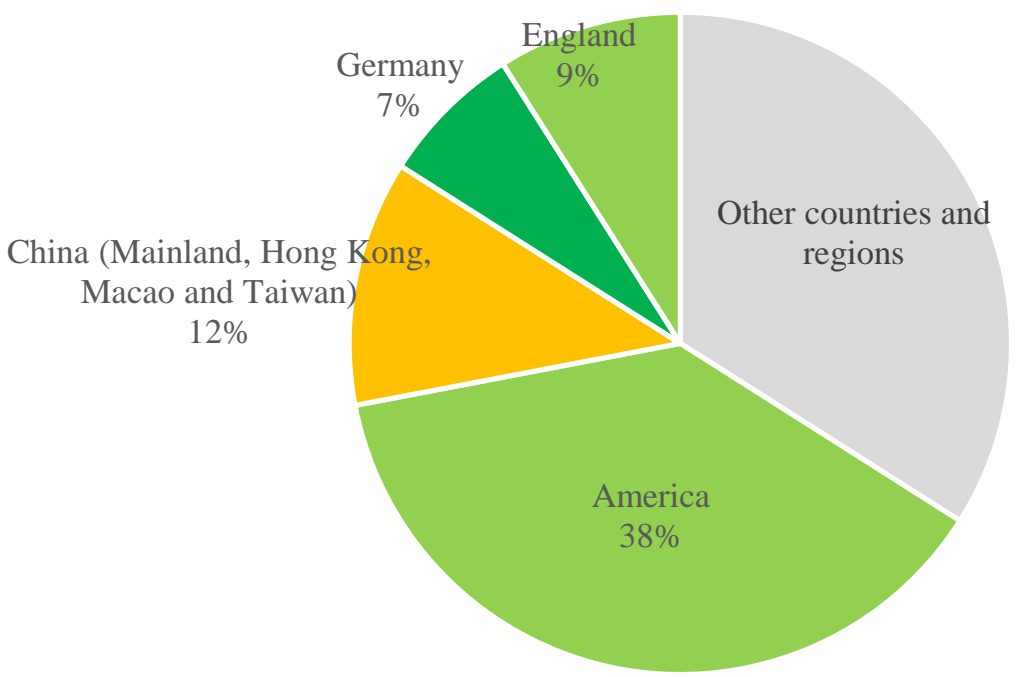

Figure 2. Top 200 scientific institutions by region distribution in the global artificial intelligence category of CSRankings 2020

\section{Main problems of artificial intelligence in promoting China's economic development}

\subsection{Lack of key and core technologies}

In the current world situation, artificial intelligence is regarded as an important new growth point of economic and social development. All countries attach great importance to the development of AI technology. China has now made great advances on the levels of innovation, optimization, and engineering implementation of AI. The research and development work on intelligent technology has been steadily advancing. Areas, such as of the theory of disruptive innovation and breakthroughs, in addition to the step 
model, are also points of interest. There is still a gap between China and developed countries in terms of key technologies, chip manufacturing, architecture design, and underlying platforms. One of the ways for AI to promote high-quality economic development is through the expansion and growth of its core industries. In the absence of the ability to speak of core technologies, China would find it difficult to pave its way in the expansion of its core industries, and even fall into a passive situation of being large but not strong for a long time.

\subsection{Privacy protection and security issues}

Compared with developed countries, China still lacks social etiquette and legal norms related to artificial intelligence system research and overall planning. This complicates the use of artificial intelligence and increases the possibility of potential risks. Potential risks include but are not limited to privacy protection, and they lack prior predicates and emergency disposal plans, especially in the field of social governance. In addition, privacy infringement and non-invasive detection of events are areas of concern. It may even go as far as causing serious mass social problems that threaten social stability and national security. Laws and regulations pertaining to artificial intelligence need to be improved, the development of the industry needs to be regulated by ethical standards, and a series of risks, such as unclear legal status, opaque algorithms, and unexplainable dangerous decisions, need to be addressed urgently. The AI industry in China has suffered damage to its international reputation and has been subjected to economic sanctions.

\subsection{Shortage of relevant talents and unreasonable structure}

The total number of high-end talents is relatively small. According to the 2020 Global AI Talent Flow Report released by Element AI, a well-known AI company, although China is at the forefront of the world in AI, China's AI talent pool is not performing well. In the global AI talent pool, the United States ranks first again with 188,300 talents, while India ranks second with 86,213 talents. China has only 22,191 AI talents, ranking fourth. Moreover, according to estimates, from 2014 to 2019, there is an increasing net outflow of AI talents from China. In addition, the talent structure is not reasonable. China's AI talents are mainly concentrated in application fields, while in the U.S., they are mainly concentrated in basic technologies, such as chips, machine learning, and computer vision. At present, there is a lack of interdisciplinary talents with the knowledge system of artificial intelligence and the real economy in China. This phenomenon criticizes the professional setting of colleges and universities, which lags behind the reality of scientific and technological development. In addition, the teaching of artificial intelligence in these colleges and universities is characterized by a lack of practice. This is reinforced by broad fields lacking the necessary specific knowledge related to AI, knowledge obsolescence using outdated methods for current problems, fragmentation, and other serious issues. The industry faces problems of flawed talent use and the lack of incentive mechanisms. The use of talents is hindered by ineffective methods to evaluate the contribution of scientific and technological personnel. Incentives for personnel can be increased by using more effective methods in the selection, appointment, training, and use of artificial intelligence for technical personnel.

\section{Conclusion}

Pushing key generic technology as well as basic theory research and development would strengthen the support of science and technology. In view of the core industry, low-quality expansion problems should give full play to the new system of national advantage. It is imperative to design productions with advantages in promoting machine learning, intelligent computing, core algorithm, as well as basic software and hardware that utilizes artificial intelligence. Lastly, key core technology research and application of 
investment should also be strengthened.

It is important to adhere to the central role of innovation in high-quality economic development, strengthen the capacity for independent innovation in science and technology, as well as enhance the capacity for scientific and technological self-reliance in the field of artificial intelligence. In order to address the issue of inefficiency and enabling the convergence of industries, it is necessary to vigorously promote open collaborative innovation with enterprises as the main body and the combination of government, industries, universities, research institutes, and applications, so as to ensure a more open and collaborative innovative ecosystem. In order to strengthen the construction of state key laboratory of artificial intelligence related enterprises, support enterprises need to cooperate with colleges, universities, research institutions, and joint laboratories, thus breaking the barrier of learning. In addition, the complications between technology, talent, system, and data flow path should be addressed. Common problems while facing artificial intelligence and applying basic research must be resolved accordingly among all stakeholders. This can be done by encouraging the innovative application of AI technologies in different industries, speeding up the coverage of AI applications, and expanding the enabling boundary of AI applications. The deep integration of the real economy and artificial intelligence should be encouraged; the dynamic and high-quality development of integrated industries should be promoted.

The new generation of artificial intelligence technology is a data-driven technology, and there is a deep contradiction between encouraging the social flow and sharing of data in technology with requiring individuals to strengthen the security protection of private information. The high quality, large scale, and availability of data are critical to the success or failure of artificial intelligence development. Therefore, in terms of data security, the government should place importance in the establishment of relevant laws and regulations, industry associations should accelerate the formulation of industry standards and norms, while enterprises should build and strictly implement punishments for data leakage. It is vital to implement the Cyber Security Law and the Data Security Law of the People's Republic of China as well as call for the early introduction of the Personal Information Protection Law of the People's Republic of China. Drawing on the EU's advanced experience in data security and privacy protection, such as the General Data Protection Regulation, relevant implementation rules and supporting policies should be issued in light of the actual situation to encourage data connectivity on the premise of ensuring data security and personal privacy concerning enterprises' secrets. In view of the problems, organization managers, legal experts, and technical experts are recommended to cooperate, the whole society is encouraged to participate in discussions, and the government is advised to formulate relevant laws, regulations, and ethics, specifically targeting artificial intelligence information. This includes collection, storage, use, share, transfer, and disclosure of information, information security, privacy, as well as the balance between data availability, to ensure that artificial intelligence technology is used for advancing the economy, society, and nation in a manner that would bring prosperity to everyone.

\section{Disclosure statement}

The authors declare that there is no conflict of interest.

\section{References}

[1] Acemoglu D, Restrepo P, 2017, Secular Stagnation? The Effect of Aging on Economic Growth in the Age of Automation. J American Economic Review, 107(5): 174-179.

[2] Jones CI, Aghion P, Jones BF, 2017, Artificial Intelligence and Economic Growth. SIEPR, (17-027): 56. 
[3] Wang Z, Feng H, 2020, Research on the Influence of Sci-Tech Service Industry Development on China's Economic Growth. Macroeconomics, (6): 102-113.

[4] Akaev AA, Rudskoi AI, 2017, Economic Potential of Breakthrough Technologies and Its Social Consequences, in Industry 4.0, Springer International Publishing, 13-41. 